THE LANCET, December 31, 1881.

\section{olimical andeture}

\section{MEDULLO-A R T H R T IS.}

BY J. GREIG SMITH, M.A., SURGEON TO THE BRISTOL ROYAL INFIRMARY.

(Concluded from p. 10\%8.)

THE two cases I now show you are intended to illustrate the treatment of medullo-arthritis. They are not exactly typical cases of the disease, being more acute and more grave than we usually meet with; but I hope they will be all the more impressive and instructive on that account.

Our first patient, a girl of fourteen, was armitted in a wretched condition some months ago. She was much emaciated, and suffered from continuous diarrhoea. She had been bedridden for some weeks with pain in her left knee, but beyond this fact we could get no trastwortby history. On examination, the joint cavity was full of fluid, and there co-existed a peri-articular abscess over the outer condyle. Her evening temperature was $103^{\circ}$. She complained of great pain in the joint. Next morning this abscess was opened, and as the pus flowed the joint also emptied itself. In our manipulations of the joint some air (antiseptic, of course) got in, and we could feel the bubbling and crackling inside. Next night the temperature wa $988^{\circ}$, and the other symptoms were somewhat improved. For three weeks the knee was dressed on every third day; but matters did not progress satisfactorily, and towards the end of the third week her evening temperatures had again risen to $102^{\circ}$. The cause of this was found to be a huge abscess over the right buttock. This was opened, dressed antiseptically three times during three weeks, and at the end of that time was perfectly healed. In the meantime her temperature had remained nearly normal, and she was gaining flesh and strength. But her temperature suddenly went up again, with pain in the other knee-joint. Rest, cotton-wool, \&c., had no influence, and there was no doubt that this knee also was suppurating. Albumen was at this time found in the urine.

It was now clear that we had to deal with a condition of pyæmia. We know that osteo-myelitis is a frequent cause of pyæmia, and we had every reason to believe that suppuration was going on inside the condyles of our patient's left femur. The indication was clear: to give a free exit to the suppurating marrow bound down inside the femur, and engaged in the meshes of its cancellous bone. The only other alternative was amputation.

Six ounces of flaky pus were removed from the right knee-joint, and two large drainage-tubes were inserted. On the left limb an opening ws made by a gouge towards the outer and posterior aspect of the outer condyle, and the con tents of both condyles were scraped out. Sufficient proof of the existence of disease was given in the fact that a Volk. mann's spoon was strong enough to break down the cancellous bone inside. With the little finger in the opening it was possible to feel the articular cartilage. A large drainagetube was inserted into the bottom of the cavity in the cóndyles.

From that time to the present, when you see the patient jump down from a chair and walk about without lameness, the course was one of uninterrupted improvement. The temperature became normal and coutinued so. Diarrhœa ceased, as did the albuminuria. In ten days, af $\mathrm{er}$ two antiseptic dressings, the wounds in the right knee-joint were closed and skinned over. Antiseptic dressiugs were applied to the left knee-joint for six weeks, when they were changed for boracic lint. The cavity inside the condyles is now filled up, and there remains only a small superficial sore. I may add that we were careful to move both joints every time we dressed them.

Our other patient is also a girl of fourteen. She came from South Wales some months ago with suppuration inside the right knee-joint, starting from osteo-myelitis of the lower end of the femur. She had been six weeks ill in bed, but had been lame for some werks previously. The whole of the right limb was swollen and in parts oedematous; there was considerable bony thickening of the lower half of the No. 3044 femur, and the joint cavity was enormously distended by fluid, which was on the point of bursting through the skin on the inner side of the patella. There existed in this case also a peri-articular abscess, produced apparently by bursting of the joint capsule. I need not say that there were much pain and starting with considerable fever. Here, then, was a case of osteo-myelitis of the end of the femur, causing an abscess, which had burst into the knee-joint. It differed from an ordinary medullo-arthritis, in extending for some distance up the femur, and from pure osteo-myelitis in being so chronic.

At first, as in the other case, we tried simple drainage of the joint. Matters were considerably improved thereby, but it was clear after three months' treatment that the real cause of the disease had not been removed. Accordingly, exactly the same operation was performed as in the pre vions case. The whole of the contents of the femoral condyles were removed through an opening made (in this case) on the inner side. After all the softened tissues had been removed, the finger introduced through the opening not only could feel the $c_{\text {xp }}$ of the articular cartilage, but could make it pout at the sides of the ligamentum patellæ. A large drainage tube was inserted and retained for six weeks. At the end of that time it was removed; antiseptics were stopped and boracic lint dressing applied. There is still some slight discharge, but this is daily diminishing, and the patient will soou, Ihope, be wa'king about. Here the result will not be so good as in the other case, on account of the adhesions which have formed. I did not dare to institute passive movement here, becuuse the articular cartilage wats left wirh so little support. And as the cartilage will give us some of the new bone destined to fill up the cavity which we made, we must be careful not to iaterfere with its nutrition more than we can help. Besides, I believe it had contracted adhesions to the tibial cartilage during the period the joint was being drained.

These, gentlemen, were argravated instances of medulloarthritis; and I call your attention specially to these as the most powerfal form of advocacy $I$ can employ in support of what I believe to be the best treatment of all cases of medullo-arthritis when simple measures of rest, counterirritation, \&e, have failed. And I would venture to recommend it specially in the early stages of the disease. In the later stages, when the soft parts are disorganised and the bones carious, we are driven to mutilation of the limbamputation or excision. "It will come to excision" is an expression very often applied to these forms of joint disease. It is an opprobrum to our art that we should so frequently have to stand by with folded hands while such cases dogeredly go on to that extent which will justify the major operation.

I bolieve that if the patholngical condition is recosnised sufficiently early the prouress of the disease might be nipped in the bud. If it is an area of inflamed marrow that is the root of the evil, and if we can reach and remove this, we ought to cure the disease. If there be any justification for the treatment of strumous glands in the neck by removal, there is much stronger justification for the removal of a similarly diseased structure inside bone. Inside a bony shell everything is against spontanenus cure. Compressed and perhaps strangulated by the outer shell the inflammatory products are driven to destruction; and, when decay his set in, the abundant bony trabecula entangle the degenerate products and delay their discharge through any opening that may have formed. The very chronicity of its course begets throughness of destruction. Long after the marrow is in a state of suppurative or careous degeneration the bony trabeculæ remain to confine it, simply because, being now almost bereft of vitality, the decayed tissues cannot cause bony absorption or disintegration. Bony caries is always a tedious complaint.

Every thing, therefore, points to a justification of the early treatment of such diseases. Spontaneous cure is so rare and the ulterior effects are so disastrous, that any operative procedure which has a sound pathological foundation does not endanger life or limh, and promises to cure the disease, is sure to be weleomed by surgeons. The tapping of a marrow cavity and scooping out more or less of the inflamed marrow and bony trabeculæ is not a severe operation, and it seems to me to be a sound one. It is probably a question more of diagnosis than anything else. If you are satisfied that chronic medullitis exists inside a bone, and you find that ordinary measures are ineffectual, I think you ought to provide an exit for the inflammatory tissues, otherwise they will 
assuredly make an exit for themselves along a channel that will almost certainly cause destruction of the articulation, or even of the limb.

The early treatment of chronic joint disease is, at the present day, one of the most pressing questions in surgery. Recent discussions on this point have dome little else beyond indicating our general therapeutic helplessness. An exception ought to be made in favour of Mr. Teale's plan of giving an exit to fluid which is distending a joint in the earlier stages of the synovial disease. By relieving tension inside an inflamed joint one continuing cause of the mischief is, for the time at least, removed. But the chief cause remains, for we find that such a simple measure is rarely sufficient to cure the disease. For the other form of disease, medullo-arthritis, I know of no early form of treatment except the universal one for all inflammations-rest. And how unsatisfactory this is, the great number of joint excisions annually performed in our large hospitals is sufficient testimony.

At another time I shall take occasion to describe to you how I would recommend you to treat cases of synovioarthritis which do not improve under treatment by simple rest. My first case, one of synovial disease of the elbowjoint with peri-articular abscesses, occurred two years ago. The ordinary incisions for excision of the joint were made, but we excised not the joint, only the disease. The articular cartilages, so much of them at least as the fungating granulations had not eaten away, were left; no bone was removed, and the girl went out with a limb much more perfect than could have resulted from the most favourable excision. And some of my coll ragues in the last two years have had similar good results. The more I see of syuovio-arthritis the more I am convinced that it ought never to become a cause for excision, still less for aroputation. Too often it has gone on to such an extent that nothing but excision or amputation will save the patient's life when it comes under our notice. But too frequently also, I am afraid that we ourselves while endeavouring to effect a cure permit the case to drift on to the major operation

In medullo-arthritis, after abscess has formed or suppurative synovitis has been set $u p$, any operative interference which is less severe than excision would realily be granted a trial. Before the joint cavity has become invaded we should be very chary of making an entrance into the medullary cavity, except on one condition. This condition is that the cavity which we make shall not suppurate. With the help of antiseptics I believe we may nearly always fulfil the condition-that is to say, we do it through the medium of organised blood-clot.

Let me relate to you the case which first convinced me that clot organisation was an end to be definitely arrived at in surgery. We had to deal with caries of the os calcis in a boy of seventeen. The contents of the bone were removed, nothing being left but the compact outer shell. Sinuses were present, so we swabhed ont the cavity with chloride of zinc. The cavity soon filled with blood; this blood was emptied out again and again, and the third filling was permitted to remain. The wound in the skin was accurately stitched up, and no means of drainage were adopted. We dressed it for the first time at the end of ten days, and then the wound was skinned over. The cavity inside the bone was filled with organising clot, and we had the carious bone replaced by a tissue which would ultimately become fibrous, and suitable as a nidus for bony development. Since then I have treated every case of caries of the bones of the foot that has come uoder my care in this way. Clot organisation has not always been got, but we have never had to excise the bone.

Now, if we can get primary union after leaving such a large cavity in bone as in the above case, and after using a powerful chemical like chloride of zinc, there is a much higher probability of our securing the same end where the cavity is smaller and there is no irritating application used. If we are assured, in any given case, that there exists an area of inflamed marrow inside a bone, I believe we may remove this area with something like certainty that the cavity we leave behind will not suppurate, and with a high probability that the disease will be cut short. Mere drilling will not do. If the trabeculæe of bone did not exist to entangle the marrow, simple tapping of the compact shell might succeed. The trephining of a mastoid abscess so frequently fails to save the patient's life, because an opening in the compact shell is not sufficient to eusure a free exit to the pus. I would advise you in mas- toid abscess, to remove by gouge as much of the cancellated bone as you possibly can; and for the same reason that I should advise it in medullitis-nannely, that the trabeculre serve to confine the inflammatory products.

The joint most commonly affected with medullo-arthritis is the hip; and it is in the early stages of hip-joint disease that the procedure I advocate would be most frequently performed. Most of you have already seen the operation, but I may again describe it. If you feel around the great trochanter of the femur you will be able to make out three promineut points or tubercles. These points are the anterior superior, the posterior inferior, and the anterior inferior angles of the trochanter. They form roughly the angles of an equilateral triangle. The point to enter the bone (which is the end of the axis of the neck of the femur) is at the junction of the anterior with the middle third of the base of this triangle. An opening in the compact bone may be made with a gouge by simply rotating and pushing Carefully keeping in the centre of the neck, we drill a cavity in the bone with a smaller gouge, or, if the bone is soft enough, with a Volkmann's scraper. As we approach the layer of epiphysial cartilage we must be careful, for inflammation may so have softened it that rough handling may break off the diaphvsis and set it lnose in the joint. We remove this cartilaginous barrier $c$ nd tap the marrow inside the diaphysis of the head. If the $b$ ne is soft here it may be scooped out; if not it ought to be left. The epiphysial cartilage, as well as the other tissues removed, will serve to give us an idea as to the amount of disease. The simple incision is carefully closed, and you leave the dressings on for ten days or so. At that time complete union will pro. bably have taken place. An exactly similar proceeding for the other bones may be readily carried out. Every surgeon must judge for himself how much bone is to be removed and where it is to be most conveniently approached.

Let me give you a few hints as to the best means of securing clot organisation; or if this term is objected to, of filling a wound cavity by blood-coagulum, which is either itself to be converted into a permanent medium of union, or is to serve as a nidus for the ingrowth of a tissue which will fulfil. that purpose. The part must be kept at perfect rest for at least twenty-four hours. If possible, coagulation should have begun before the wound is closed. If a desepticising agent, such as chloride of zinc, has been used, the first two or three fillings with blood should be emptied out. If not enough blood is got, a fresh wound may be made in the areolar tissue. Do not use a drainage-tube. Its removal disturbs the clot and leaves a cavity, the sides of which are liable to break down or suppurate. Horsehair drains may be used; the presence of a few horsehairs distributed through the clot may help coagulation, and, gathered together in a bundle at the end of the wound, they may provide for the exit of any superfluous serum.

In conclusion, I would venture to recommend this treatment of the early stages of morbus coxæ and allied diseases, not as a substitute for the milder measures of rest, counterirritation, and such-like, but as a possible preventive of excision or amputation. How far it is destined to be suc. cessful experience alone can tell. The small trial which I have been able to give it has afforded me results either entirely favourable or negative. It has done no harm. Cases suitable for the operation are few. When the patient comes for treatment early enough we often get spontaneous cure, but cure, be it noted, nearly always with stiff joint. Would our operation prevent anchylosis if performed soon enough? More frequently the case turns up too late, when abscesses have formed and the articular surfaces are destroved, so that no course is left but excision or amputation. We cannot expect, even in a minority of instances, such a favourable result as in our first case. The fact that such a result may be got is the strongest argument I can put forward in support of the uperation.

Presentatrons. - Dr. Taaffe, physician at the Royal Alexandra Hospital for Sick Children, Briyhton, was on the 24th inst. presented with a gold watch, suitably inscribed, with a cheque for $£ 220$, in recognition of his services in connexion with the erection of the new building. The Mayor made the presentation. - - The Guardians of the Upton-on-Severn Union have presented Mr. Wadams, late medical officer for the Malvern district, with a cheque for

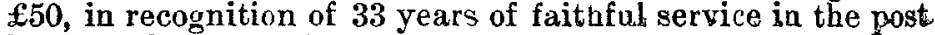
he recently resigned. 